\title{
Engineering Design Education and Time Management
}

\author{
W. Ernst Eder \\ Professor Emeritus \\ eder-e@kos.net
}

\begin{abstract}
A brief historic view of design engineering shows the roles of problem definition (design specification), conceptualizing, estimations, layouts, detail and assembly drawings, parts lists, etc., to define an engineering product (process or tangible system according to ISO9000:2005) for implementation and/or manufacture and use. A brief assessment of computer-aided design (CAD) over the last 40 years reveals its detrimental effects on design engineering the previously usual (intuitive) conceptualizing and preparation of layouts has been neglected. Design engineering is compared to the artistic design disciplines, and differences are highlighted. Design engineering must apply the constraints imposed by the engineering sciences, to satisfy customer requirements, to consider economics, and to conform to laws and regulations. Yet design engineering offers the opportunity to use several more abstract representations of technical processes and technical systems to aid conceptual designing. A role is indicated for design methods and systematic approaches for design engineering, especially for design and redesign for innovations (non-routine tasks), and in engineering education. Parts of the Theory of Technical Systems and Engineering Design Science are outlined, with conesquences for proposal of a theory-based systematic design method. Especially, the role of problem solving as a sub-process in designing shows the need for iterative and recursive procedure. Design engineering demands that sufficient time is available for reflective thought, and needs good time management for any task, especially where innovation is expected. Engineering sciences are shown as necessary, but not sufficient. A broader context needs to include instruction in time management, and should be developed during engineering education in all branches.
\end{abstract}

\section{Introduction}

Time management has for many decades been important for engineering. Its importance for modern engineering education needs to be emphasized.
In the era known as B.C. (before computers), the process of design engineering (especially for industrial equipment) had an expected output in the form of detail drawings of all constructional parts prepared on translucent tracing paper (or cloth) to relevant national standards, including tolerances, surface finish, raw materials, etc. under consideration of likely manufacturing methods. In addition, assembly drawings, parts lists, assembly and adjustment instructions, usage instructions, repair instructions, etc. were prepared. Checking of these documents was always performed to ensure accuracy and completeness.

For a novel product (usage or transformation process, TrfP, and/or technical system, TS), a senior enginee-ring designer (usually a University graduate) would conceptualize, produce sketch layouts, perform preliminary calculations of expected performance and capabilities, and produce a final layout to correct sizes, e.g. using drafting machines. Technologists would then produce the detail drawings, and a checkassembly. Specialized checking personnel checked the drawings. Similar tasks needed to be completed for redesign but with much less conceptualizing.

This was obvious at the time, but needs to be repeated for the current situation in which much of the previous information has been lost.

Engineering designers can obviously design without computers. Even when designing with computers, designers often need to do some preparation work without computer assistance. Computers cannot design completely independently, generally computers are tools to assist designing. Some parts of designing may be automated. Computers help to solve problems, contribute to improvements in $\operatorname{TrfP}(\mathrm{s})$ and/or TS(s), optimize quality of the product, and improve the parameters of the design process - the addition of '(s)' signifies that product that should be designed.

Acceptance by industry of early 2-D and 3D-CAD applications (due to their limitations) caused a drastic change in detail-design procedures. CAD applications could not be used for layouts, most of them are still not suitable. Detail design of individual constructional parts tended to be allocated to different designers on their 'own computer seat'. Coordination among these 
specialists became difficult, and many errors resulted. The latest versions of some CAD applications are starting to allow 'inheritance' of some properties from one constructional part to another, and automated check assembly, see figure 1 [1].

Figure 1 Progress of Computer Support for Design Engineering (Adapted from [1] with permission)

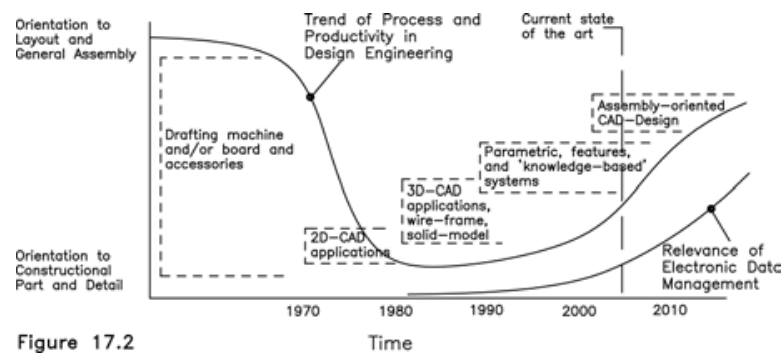

\section{Comparison of Artistic Design to Design Engineering}

If a product is intended to be visually attractive and user-friendly, its form (especially its observable shape) is important $-\mathrm{a}$ task for industrial designers, architects and similar professionals. Industrial design $[2,3,4]$, in the English interpretation, primary for consumer products and durables, emphasizes the artistic elements, appearance, ergonomics, marketing, customer appeal, satisfaction, and other observable properties of a product. This includes colour, line, shape, form, pattern, texture, proportion, juxtaposition, emotional reactions [5], etc. The task given to or chosen by industrial designers is usually specified in rough terms. The mainly intuitive design process with emphasis on 'creativity' and judgement, is used in architecture, typographic design, fine art, etc.

A major difference between industrial design and design engineering is interpreting 'conceptualizing'. For industrial designers, conceptualizing for possible future tangible products consists of preliminary sketches of observable possibilities - a direct entry into hardware (constructional structure) and its representation. The sketches are progressively refined, and eventually 'rendered' (drawn and coloured, and/or modeled by computer or in tangible materials) into visually assessable presentation material, full artistic views of the proposed artifact, to provide a 'final' presentation, for management approval. Louridas [6] describes such designers as bricoleurs [7] or tinkerers who collage divergent ideas to form a product. Considerations of engineering take place if necessary, but often at a rudimentary level. Industrial design usually works 'outside inwards', defining the envelope of observable properties, thus constraining the internal constituents and actions.

If a tangible product should work, fulfill a purpose, help to perform a process (e.g. mechanical, chemical, electro-magnetic), its functioning and operation are important - a task for engineering designers across the engineering disciplines - anticipating and analyzing this functioning is the role of the engineering sciences. Engineering intends to create what does not yet exist, that is likely to work. Engineering needs designers to be aware of a wide range of existing information and its complex interactions, and to accommodate all possible influences of scientific, technical, economic, societal, political and other areas to achieve a successful and optimal product.

Engineering designers tend to be primary for technical systems, TS(s), and their operational (usage, transfor-mation) processes, $\operatorname{TrfP}(\mathrm{s})$, as well as their manufacturing processes. These designers solve problems of making something work, manufacturability, economics, and life-cycle related properties. They work from critical zones for capability of functioning, e.g. form-giving zones, from 'inside outwards', defining the internal operational means first, constraining the outside. Novelty may be a consideration, but primary considerations are reliability (risk control), operational safety, and achievability of operation, performance and behaviour, by establishing the elemental, intrinsic, and general design properties, to indirectly achieve the observable properties.

Engineering designers develop their own systematic approaches to their range of design tasks and products - even though they usually cannot elucidate or explain these approaches and methods. For engineering education, a theory-based explainable, systematic and methodical approach can be effective to reduce the time taken to learn designing.

Engineering designers often work on routine tasks, about $95 \%$ of all design engineering is routine. When difficulties arise, these designers must resort to risk operation [8], or even safety operation, when their experience is no longer reliable or sufficient. Then, they must use an effective set of instructions, an explicit method, to overcome their problem, and increase their expertise [9]. The necessary method(s) need(s) to be learned in advance of such critical use - trying to learn a method 'on the job' is a recipe for failure - learning is best achieved in a low-threat environment, with explanation of reasons and theoretical basis, demonstration, and supervised practice.

A Chinese wisdom credited to Confucius says: Tell me and I will forget Show me and I will remember Involve me and I will understand Take one step back and I will act.

As separate statements, the first two items tend to 
deny the effectiveness of lectures and demonstrations, and to advocate only project-based and/or problembased learning [10]. The last item is usually omitted. These statements are best interpreted in combination:

Do all four and I will become competent.

This form of experiential learning takes time and effort from both the learner and the mentor/teacher time that needs to be managed for greatest effectiveness (but not necessarily efficiency). Exposure should be parallel with learning the engineering sciences, and permeate all years of instruction. The learner needs to 'hear, read, mark, learn and inwardly digest' - inward digestion consists of (a) learning well enough that the original methodical instructions are no longer absolutely necessary, and can eventually be denied by the used, and (b) making mental associations with other items that the learner knows, obtaining better context and understanding. Neither of these can be effectively tested in a teaching/learning situation by conventional examinations, but both take time and effort to achieve with the help of guiding and mentoring instruction.

\section{Systematic Design Engineering}

All engineering education should therefore provide a systematic design method for novices to learn preferably theory-based - plus appropriate 'industrial best practice' pragmatic methods. Such a theory-based method has been developed since about 1965 [11-17]. The concept of the system of Engineering Design Science (EDS) [15-17] is based on the triad 'theory subject - method', formulated in cybernetics [18]: 'both theory and method emerge from the phenomenon of the subject'. A close relationship should exist between a subject (its nature as a concept or product), a basic theory (formal or informal, recorded or in a human mind), and a recommended method for voluntary application. A theory about a subject allows a method to be defined and heuristically applied, for using or for designing the subject.

Knowledge and information about design engineering can be 'mapped' onto two orthogonal axes. The north-south axis ranges from 'practice information' to 'theory knowledge', the west-east axis ranges from 'information about existing transformation systems' to 'information bout designing and design processes'. Important are (a) information about technical and other objects, existing and to be designed, and (b) information about design processes, the mental activities and methods that may be useful.

The basis of the theory of technical systems $[12,14$ 17] is an abstract model applicable for all (artificial, man-made) transformation systems, see figure 2 . This model of the transformation system (TrfS) states:
Figure 2. General Model of a Transformation System $[16,17]$

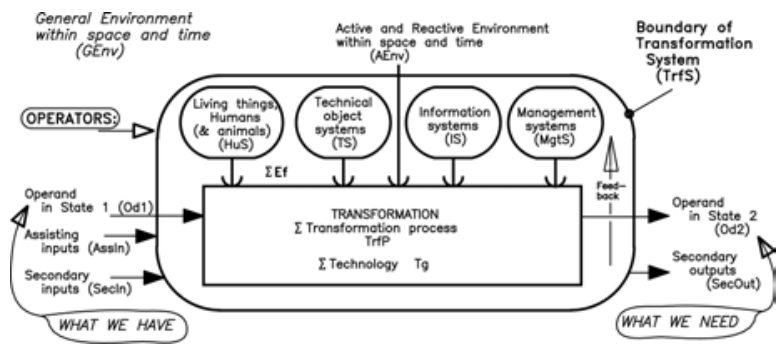

- An operand (materials, energy, information, and/or living things - M, E, I, L) in state Od1 is transformed into state Od2, using the active and reactive effects (in the form of materials, energy and/or information - M, E, I) exerted continuously, intermittently or instantaneously by the operators (human systems, technical systems, active and reactive environment, information systems, and management systems, as output effects from their internal and cross-boundary processes, acting separately and/or jointly), by applying a suitable technology $\mathrm{Tg}$ (which mediates the exchange of $M, E$, I between effects and operand), whereby assisting inputs are needed, and secondary inputs and outputs can occur for the operand and for the operators.

The general environment (regional, national and global) covers physical, chemical, societal, economic, cultural, political, ideological, geographic and all other influences indirectly acting on or reacting to the TrfS. A generalized life cycle of technical systems, TS(s), consists of typically seven (classes of) transformation systems: product planning, designing, preparation for the subsequent life-cycle phases and their supply chains, manufacture and assembly, distribution, operation of the product, liquidation and disposal.

Classes of properties of existing transformation processes and technical systems are separated into observable, mediating, and elemental design properties. TS-observable are the properties that anyone can see, assess and/or measure for the chosen TSboundaries. TS-mediating include the properties that are hidden by the chosen TS-boundaries, those related to engineering experiential information (intrinsic properties, e.g. mode of action) and engineering sciences (general design properties). TS-elemental design properties are those that are under the direct control of engineering designers during designing, and include the TS-structures (function, organ and constructional, see figure 3), their elements, classes of arrangement, form, size, materials, anticipated manufacturing methods, deviations, surface quality, etc. A 
Figure 3. Model of a Technical System - Structures $[16,17]$

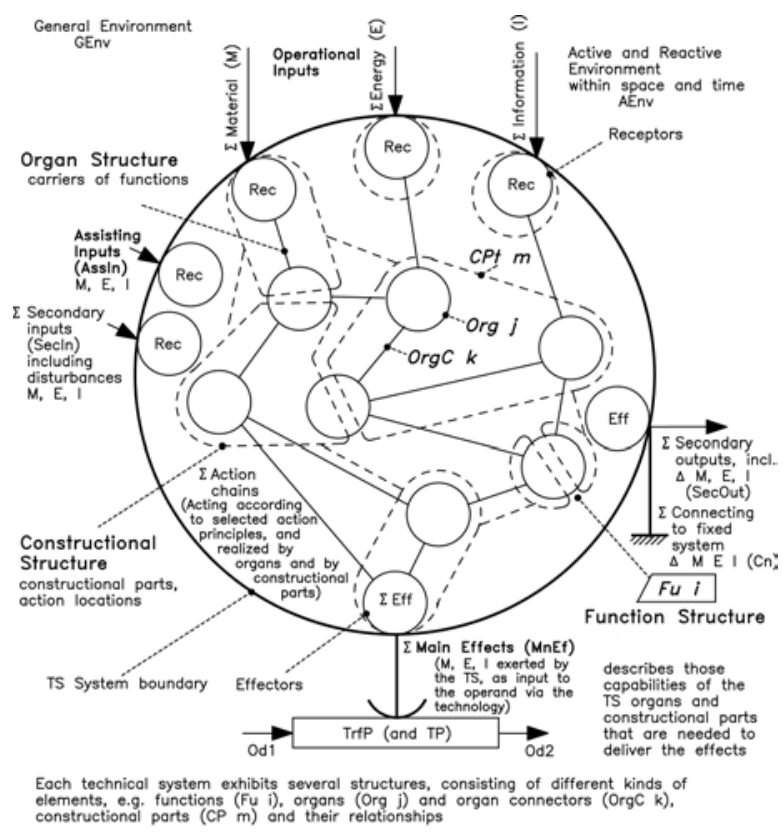

property can appear in one or more classes, and the classification can change according to the situation, e.g. a change of the assumed TS-boundaries.

Once the logic of the transformation system (TrfS), figure 2, and of the structures of TS, figure 3 , is understood, it is easily possible to derive a systematic approach to design engineering of transformation systems as products - TrfP and TS[12-17].

Normally, innovations are limited to a relatively small section of a product, partly to limit risks, partly to bring a 'new' product to market as quickly as possible. Case examples showing of this systematic approach, using the models from the theory of technical systems [12,16,17], are available in $[13,16,17]$.

Superimposed on design engineering is a frequently applied sub-process of problem solving (first defined in [12]). In its latest manifestation, this problem solving process appears as in figure 4 . Noteworthy are three auxiliary processes: Op-H3.5 'Prepare information', Op-H3.6 'Verify, check, reflect', and Op-H3.7 'Represent' - these are not specifically stated in any other model of problem solving.

The main cycle, Op-H3.1-Op-H3.4 has recently been confirmed in cognitive psychology under the desig-nation of 'creativity', with phases of 'analysis', 'generation', 'evaluation', and 'communication/implementation' - the identity is obvious, and creativity resides mainly in Op-H3.2 and 'generation'.

The statements by Wallas [27] in figure 4 cannot be over-emphasized. In problem solving (and conesquently in design engineering) there is always a need for incubation time and opportunity, including forming mental associations - a need for time management. This implies that for learning and for designing, the activity should be started as early as possible, and be consistently followed (continuous engagement, with suitable interruptions for reflection [22,23] and incubation) throughout the problem period. Only in this way can understanding and creativity be expected, including a reasonably (and probably systematic) complete search for alternative candidate solutions.

There is almost always a need for iteration and recursion [24]. Iteration involves repeated traverse of a section of the design process in a convergent approach to the required results. Recursion involves breaking the problem down into more restricted constituents, solving these separately, and combining the results to a more holistic solution proposal. Analysis of a proposal usually leads to a single (set of) answer(s) [19]. Synthesis (design engineering) is much more than a reversal of analysis, it usually requires a search for alternative solution proposals. During design engineering, the elemental design properties (including all TS-structures) are gradually established from the requirements, in a frequently and rapidly repeated process of problem solving, with multiple iterations, and therefore usually unpredictable time needs. A relationship of these operations with the TS-properties and requirements is shown in figure 6 [17].

The architectural practice of the 'charrette', a lastminute crash effort to solve the problem in all-night group sessions, is therefore not likely to produce a best solution for the design problem. Equally, 'cramming' for an examination is a very ineffective way of learning, especially because it allows no time for reflection [22,23], incubation, maturing, and discovering context-forming mental associations.

\section{Closure}

Engineering education needs to prepare its graduates for entry into practice, especially where they are likely to be involved in designing new and revised consumer or industrial products. A systematic and methodical design procedure should be a significant subject during the whole educational program: novel procedure, redesign procedure, and problem solving.

The presentation of design engineering should provide information on background (theory, in lectures or readings), worked case examples, mentoring (several design tasks in all years of study), and then a more comprehensive capstone experience of designing (as distinct from research), to enable the start of a student-progression from novice towards competence and experience - including knowing about objects and about design processes. 
Figure 4. Basic Operations - Problem Solving in the Design Process [16,17,20-27]

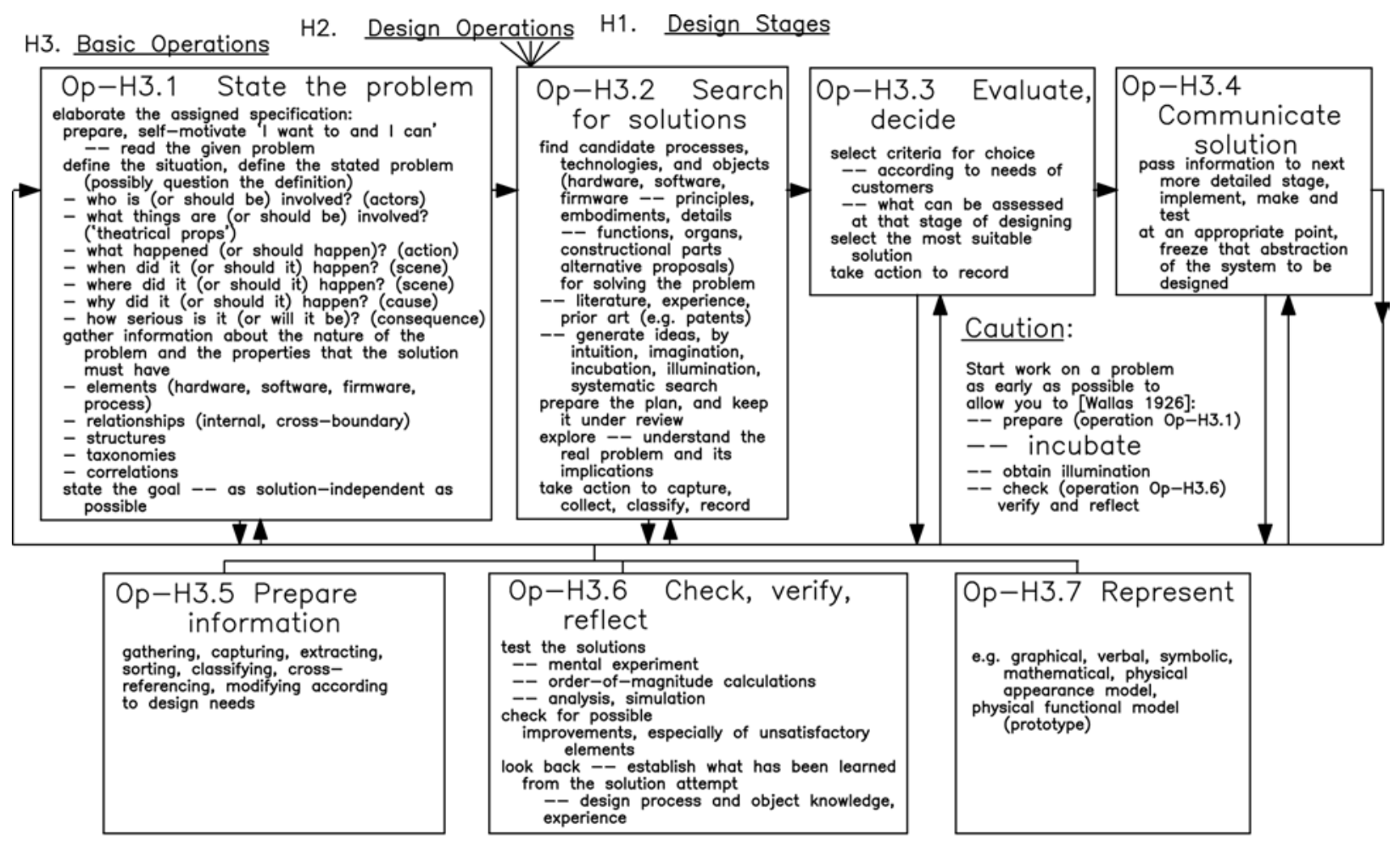

H4. Elemental Activities H5. Elemental Operations $W$

Figure 6. Main relationship Between Problem Solving, and TS-Internal and TS-External Properties (adapted from [17])

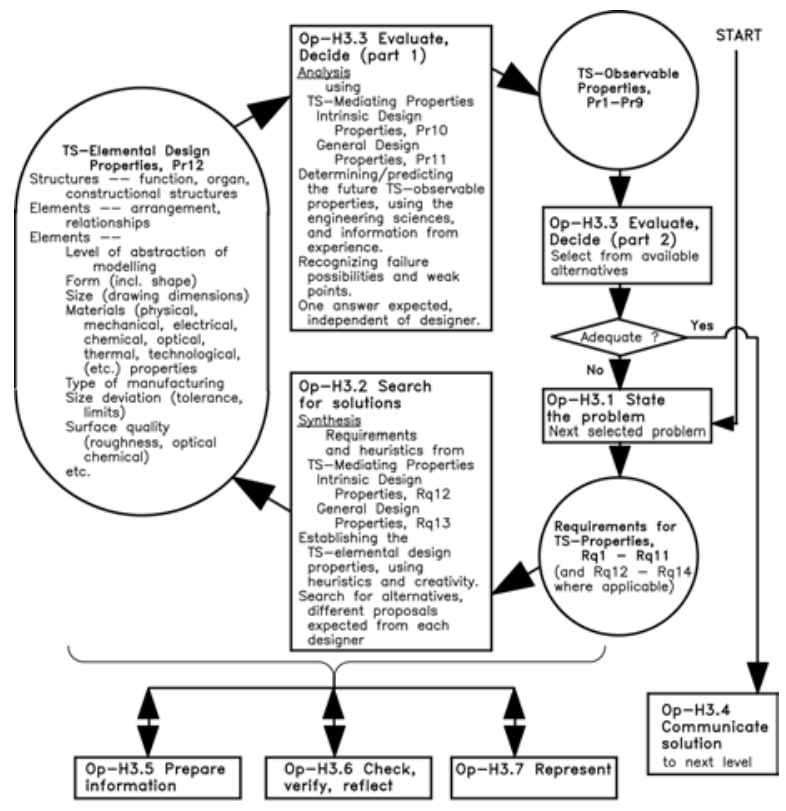

This should prepare students to handle novel and unexpected situations (when systematic progress is needed), and apply good time management

\section{References}

[1] Burr, H., Vielhaber, M., Deubel, T., Weber, C. and Haasis, S., 'CAx/engineering Data Management Integration: Enabler for Methodical Benefits in the Design Process' Jnl. Eng. Design, Vol. 16, No. 4, August 2005, p. 385-398

[2] Flurscheim, C.H. (1983) Industrial Design in Engineering: a marriage of techniques, London: The Design Council and Berlin/Heidelberg: SpringerVerlag

[3] Julier, G. (2000) The Culture of Design, London: Sage Publ.

[4] Tjalve, E., Andreasen, M.M. and Schmidt, F.F. (1979) Engineering Graphic Modelling, London: Butterworths

[5] Green, W.S. and Jordan, P.W. (2002) Pleasure With Products: Beyond Usability, Boca Raton: CRC Press 
[6] Louridas, P. (1999) 'Design as Bricolage: Anthropology Meets Design Thinking', Des. Stu., Vol. 20 No. 6, November, p. 517-535

[7] Bucciarelli, L.L. (1994) Designing Engineers, Cambridge, MA: MIT Press

[8] Müller, J., Arbeitsmethoden der Technikwissenschaften - Systematik, Heuristik, Kreativität (Working Methods of Engineering Sciences, systematics, heuristics, creativity), Berlin: Springer-Verlag, 1990

[9] Eder, W.E. (2009) 'Why Systematic Design Engineering?', paper ASME DETC2009-86067, in Proc. $6^{\text {th }}$ Symposium on International Design and Design Education, DEC 6, August 30 - September 2, 2009, San Diego, California, USA

[10] Eder, W.E. (1998) 'Einstein Got it Wrong (for once) - Some Consequences for Problem-based Learning', in Proc ASEE Annual Conference, Educational Research and Methods Division, Seattle, WA, 28 June - 1 July 1998, Washington, DC: ASEE, (CD-ROM)

[11] Hubka, V. (1976) Theorie der Konstruktionsprozesse (Theory of Design Processes), Berlin: Springer-Verlag

[12] Hubka, V. and Eder, W.E. (1988) Theory of Technical Systems: A Total Concept Theory for Engineering Design, New York: Springer-Verlag

[13] Hubka, V., Andreasen, M.M. and Eder, W.E. (1988) Practical Studies in Systematic Design, London: Butterworths - English edition of (1980) WDK 4 - Fallbeispiele

[14] Hubka, V. and W.E. Eder (1992) Engineering Design, Zürich: Heurista

[15] Hubka, V., \& Eder, W.E. (1996) Design Science: Introduction to the Needs, Scope and Organization of Engineering Design Knowledge, London: Springer-Verlag, , http://deseng.ryerson.ca/DesignScience/

[16] Eder, W.E. and Hosnedl, S (2008) Design Engineering: A Manual for Enhanced Creativity, Boca Raton: CRC-Press
[17] Eder, W.E. and Hosnedl, S (2010) Introduction to Design Engineering: Systematic Creativity and Management, Leiden, NL: CRC Press/Balkema

[18] Klaus, G. (1965) Kybernetik in philosophischer Sicht (Cybernetics in Philosophical View) 4th edn., Berlin: Dietz Verlag, 1965

[19] Eder, W.E. (2009) 'Analysis, Synthesis and Problem Solving in Design Engineering', paper 2-23, session W3 SIG-AEDS, in Proc. International Conference on Engineering Design, ICED 09, August 24 - 27, Stanford University, Stanford, 2009, California, USA

[20] Gregory, S.A. (1966) 'Design Science', in Gregory, S.A. (ed.), The Design Method, London: Butterworths, p.323-330, 1966

[21] Koen, B.V. (2003) Discussion of The Method: Conducting the Engineer's Approach to Problem Solving, New York: Oxford Univ. Press

[22] Schön, D.A. (1983) The Reflective Practitioner: How Professionals Think in Action, Basic Books, New York

[23] Schön, D.A. (1987) Educating the Reflective Practitioner: Towards a New Design for Teaching and Learning in the Professions, San Francisco: Jossey-Bass, 1987

[24] Eder, W.E., 'Requirements to Properties Iterative Problem Solving', in Proc. Canadian Engineering Education Association 2010 Inaugural Conference, 7-9 June 2010, Queen's University, Kingston, ON

[25] Wales, C.E., Nardi, A.H. and Stager, R.A. (1986a) Professional Decision-Making, Morgantown: Center for Guided Design (West Virginia Univ.)

[26] Wales, C., Nardi, A. and Stager, R. (1986b) Thinking Skills: Making a Choice, Morgantown, WV: Center for Guided Design (West Virginia Univ.)

[27] Wallas, G. (1926) The Art of Thought, London: Cape (reprint 1931) - p. 79-96 reprinted in Vernon, P.E. (ed.), Creativity, Harmondsworth: Penguin, 